\title{
ON COMPACTIFICATION OF MAPPINGS
}

\author{
by P. A. FIRBY \\ (Received 4th May 1973)
}

If $X$ and $Y$ are Tychonoff spaces then the continuous function $f$ mapping $X$ onto $Y$ is said to be compact (perfect, or proper) if it is closed and point inverses are compact. If $h$ is a continuous function mapping $X$ onto $Y$ then by a compactification of $h$ we mean a pair $\left(X^{*}, h^{*}\right)$ where $X^{*}$ is Tychonoff and contains $X$ as a dense subspace, and where $h^{*}: X^{*} \rightarrow Y$ is a compact extension of $h$. The idea of a mapping compactification first appeared in (7). In (1) it was shown that any compactification of $X$ determines a compactification of $h$, and that any compactification of $h$ can be determined in this way. This idea was then developed in (2) and (3).

Throughout this paper all topological spaces are assumed to be Tychonoff. We consider throughout a fixed continuous function $f$ mapping $X$ onto $Y$.

A compactification of $X$ is a compact Hausdorff space containing as a dense subspace a homeomorphic image of $X$. We assume $X$ to be a subspace of each of its compactifications. If $a$ is a partition of $\beta X$, the Stone-Cech compactification of $X$, then $a$ is said to be an upper semicontinuous (u.s.c.) decomposition of $\beta X$ if

(a) the members (blocks) of $a$ are compact subsets of $\beta X$ and

(b) whenever $V$ is open in $\beta X$ and contains the block $A$ of $a$, there exists an open set $W$ in $\beta X$ such that $A \subseteq W \subseteq V$ and $W$ contains any block of $a$ that it meets. (Notice that since $\beta X$ is normal the partition $a$ of $\beta X$ is u.s.c. if and only if the associated canonical quotient mapping is closed.) Refinement of partitions imposes a natural partial order upon the set of u.s.c. decompositions of $\beta X: a \leqq b$ if and only if given $A \in a$, there exists $B \in b$ such that $A \subseteq B$. The u.s.c. decompositions of $\beta X$ with this partial order form a complete lattice

(4). For our purposes we need only note that

$$
\bigwedge\left\{a_{i}: i \in I\right\}=\left\{\bigcap\left\{A_{i}: i \in I\right\}: A_{i} \in a_{i}, i \in I\right\} .
$$

A natural partial order also exists on the set of compactifications of $X$. We say $a X \leqq b X$ if and only if there exists a continuous function $h$ mapping $b X$ onto $a X$ such that $h \mid X$ is the identity. The compactifications $a X, b X$ are said to be equivalent if $a X \leqq b X$ and $b X \leqq a X$ and we consider equivalent compactifications to be the same compactification. Similarly, if $\left(X^{*}, f^{*}\right)$ and $\left(X^{\prime}, f^{\prime}\right)$ are compactifications of $f$, we say $\left(X^{*}, f^{*}\right) \leqq\left(X^{\prime}, f^{\prime}\right)$ if and only if there is a continuous function $h$ mapping $X^{\prime}$ onto $X^{*}$ such that $h \mid X$ is the identity and such that $f^{\prime}=f^{*} . h$. Again we say that $\left(X^{*}, f^{*}\right)$ and $\left(X^{\prime}, f^{\prime}\right)$

$$
\text { E.M.S. }-19 / 2-\text { H }
$$


are equivalent if $\left(X^{*}, f^{*}\right) \leqq\left(X^{\prime}, f^{\prime}\right)$ and $\left(X^{\prime}, f^{\prime}\right) \leqq\left(X^{*}, f^{*}\right)$, and by considering equivalent compactifications to be the same compactification we have a partial order defined on the compactifications of $f$. In (6) it was shown that there is a natural bijection between the set of compactifications of $X$ and the set of u.s.c. decompositions of $\beta X$ which contain the points of $X$ as blocks. If $a$ is a u.s.c. decomposition of $\beta X$ with this property then we denote by $a X$ the corresponding compactification of $X$ and by $q_{a}$ the corresponding mapping from $\beta X$ onto $a X$. (In fact $q_{a}$ is the canonical quotient mapping associated with the partition a.) In (5) it was shown that $a X \leqq b X$ if and only if $b \leqq a$.

We denote by $f: \beta X \rightarrow \beta Y$ the continuous extension of $f: X \rightarrow Y \subseteq \beta Y$, and by $u$ we mean the u.s.c. decomposition $\left\{f^{-1}(y): y \in \beta Y\right\}$ of $\beta X$. If $a$ is a u.s.c. decomposition of $\beta X$ containing the points of $X$ as blocks, and if $a \leqq u$, then denote by $p_{a}$ the restriction of $q_{a}$ to $f^{-1}(Y)$. We then define $f_{a}$ and $f_{a}^{*}$ by

$$
\begin{aligned}
f_{a} & =\bar{f} \cdot q_{a}^{-1}: a X \rightarrow \beta Y \\
f_{a}^{*} & =\bar{f} \cdot p_{a}^{-1}: f_{a}^{-1}(Y) \rightarrow Y .
\end{aligned}
$$

It is a routine matter to show that $f_{a}$ and $f_{a}^{*}$ are continuous functions onto $\beta Y$ and $Y$ respectively. Notice that $f_{a}^{-1}(Y)=q_{a}\left(f^{-1}(Y)\right)=p_{a}\left(f^{-1}(Y)\right)$ and $f_{a}^{*}$ is the restriction of $f_{a}$ to this set. Notice also that $f_{a}$, being a continuous function defined on a compact space, is compact.

Theorem 1. If aX is a compactification of $X$ then $\left(f_{a \wedge u}^{-1}(Y), f_{a \wedge u}^{*}\right)$ is a compactification of $f$ and each compactification of $f$ can be described in this way.

Proof. $a \wedge u$ is a u.s.c. decomposition of $\beta X$ containing the points of $X$ as blocks and $a \wedge u \leqq u$. Then $f_{a \wedge u}^{*}$, being the restriction of $f_{a \wedge u}$ to $f_{a \wedge u}^{-1}(Y)$ is a compact mapping, and moreover $f_{a_{\wedge} u}^{*}$ is an extension of $f$, since for each $x \in X$ $p_{a \wedge u}^{-1}(x)=x$.

Suppose now that $\left(X^{\prime}, f^{\prime}\right)$ is a compactification of $f$. Then $\beta X^{\prime}$ is a compactification of $X$ which we denote by $b X$. If $f^{\prime}: \beta X=b X \rightarrow \beta Y$ denotes the continuous extension of $f^{\prime}$, then since $\bar{f}^{\prime} \cdot q_{b}$ is equal to $f$ on $X, f^{\prime} \cdot q_{b}=f$ on $\beta X$ and so $b \leqq u$. Then $f_{b}$ is defined and, again since the extension of $f$ to $b X$ is unique, $f_{b}=f^{\prime}$ on $b X$, and so if we can show that $f_{b}^{-1}(Y)=X^{\prime}$ then the proof is complete. Suppose then that $x \in \beta X^{\prime} \backslash f^{\prime-1}(y)$. Then since $f^{\prime-1}(y)$ is compact $x$ has a compact neighbourhood $N$ disjoint from $f^{\prime-1}(y)$. Then $N \cap X^{\prime}$ is closed and non-empty and so $f^{\prime}\left(N \cap X^{\prime}\right)$ is closed, non-empty, and does not contain $y$. Then since $f^{\prime}$ is continuous

$$
f^{\prime}\left(\operatorname{cl}\left(N \cap X^{\prime}\right)\right) \subseteq \operatorname{cl}\left(f^{\prime}\left(N \cap X^{\prime}\right)\right)=f^{\prime}\left(N \cap X^{\prime}\right)
$$

and so $\bar{f}^{\prime}(x) \neq y$. Hence $X^{\prime}=f^{\prime-1}(Y)=f^{\prime-1}(Y)=f_{b}^{-1}(Y)$ and this completes the proof.

Notice that if $\left(X^{*}, f^{*}\right)$ is a compactification of $X$ then the content of Theorem 1 is to allow us to consider $X^{*}$ to be a subspace of any compactification $a X$ producing $f^{*}$. 
Theorem 2. If $\left(X^{*}, f^{*}\right)$ and $\left(X^{\prime}, f^{\prime}\right)$ are compactifications of $f$ then

$$
\left(X^{*}, f^{*}\right) \leqq\left(X^{\prime}, f^{\prime}\right)
$$

if and only if $\beta X^{*}=b X \leqq a X=\beta X^{\prime}$.

Proof. We saw in the proof of Theorem 1 that $\left(X^{*}, f^{*}\right)$ and $\left(X^{\prime}, f^{\prime}\right)$ respectively are determined by $b X$ and $a X$.

If $b X \leqq a X$ then $h$ defined by $h=p_{b} \cdot p_{a}^{-1}$ is a function onto $X^{*}$ and since $p_{a}$ is continuous and $p_{b}$ is closed, $h$ is continuous. But then

$$
f^{\prime}=\bar{f} \cdot p_{a}^{-1}=f^{*} \cdot p_{b} \cdot p_{a}^{-1}=f^{*} \cdot h .
$$

Conversely, if $\left(X^{*}, f^{*}\right) \leqq\left(X^{\prime}, f^{\prime}\right)$ then there is a continuous function $h$ mapping $X^{\prime}$ onto $X^{*}$ and such that $h \mid X$ is the identity. If we extend $h$ to the continuous function $\bar{h}: \beta X^{\prime}=a X \rightarrow b X=\beta X^{*}$ then $\bar{h}$ maps onto $\beta X^{*}$ and so $b X \leqq a X$.

If $p$ is a partition on the set $S$ and if $A \subseteq S$ then by $p \mid S$ we mean the partition $\{P \cap A: P \in p\}$.

Corollary 3. If the compactifications $\left(X^{*}, f^{*}\right)$ and $\left(X^{\prime}, f^{\prime}\right)$ of $f$ are produced by the compactifications $a X$ and $b X$ respectively, then $\left(X^{*}, f^{*}\right) \leqq\left(X^{\prime}, f^{\prime}\right)$ if and only if

$$
b \wedge u\left|f^{-1}(Y) \leqq a \wedge u\right| f^{-1}(Y) .
$$

Proof. $\left(X^{*}, f^{*}\right)$ is produced by the compactifications $a \wedge u X$ and $c X=\beta X^{*}$ and thus $a \wedge u\left|f^{-1}(Y)=c\right| f^{-1}(Y)$. Similarly, $b \wedge u\left|f^{-1}(Y)=d\right| \bar{f}^{-1}(Y)$ where $d X=\beta X^{\prime}$. Then $\left(X^{*}, f^{*}\right) \leqq\left(X^{\prime}, f^{\prime}\right)$ if and only if $c X \leqq d X$ and this is so if and only if $d \leqq c$. However, since $c X=\beta X^{*}, c$ is the minimal u.s.c. decomposition of $\beta X$ such that $c\left|f^{-1}(Y)=a \wedge u\right| f^{-1}(Y)$ and similarly $d$ is minimal such that $d\left|f^{-1}(Y)=b \wedge u\right| f^{-1}(Y)$. Thus $d \leqq c$ if and only if $b \wedge u\left|f^{-1}(Y) \leqq a \wedge u\right| f^{-1}(Y)$.

Corollary 4. The compactifications $a X, b X$ determine equivalent compactifications of $f$ if and only if in $f^{-1}(Y)$ the partitions

$$
\left\{q_{a}^{-1}(t) \cap f^{-1}(y): t \in a X, y \in Y\right\}
$$

and $\left\{q_{b}^{-1}(t) \cap f^{-1}(y): t \in b X, y \in Y\right\}$ are equal.

Corollary 5. The compactifications of $f$ with the usual partial order form a complete upper semilattice. They form a complete lattice if and only if for some compactification $s X$ of $X, s \wedge u\left|\left(f^{-1}(Y) \backslash X\right)=u\right|\left(f^{-1}(Y) \backslash X\right)$.

Proof. If $\left\{\left(X_{i}, f_{i}\right): i \in I\right\}$ is a family of compactifications of $f$ then

$$
\left\{b_{i} X=\beta X_{i}: i \in I\right\}
$$

being a family of compactifications of $X$, has a supremum, $a X$ say. Then for each $i \in I, a \leqq b_{i} \leqq u$ and so from Corollary 3 we see that $\left(X_{a}^{*}, f_{a}^{*}\right)$ is an upper bound for $\left\{\left(X_{i}, f_{i}\right): i \in I\right\}$. Moreover, if $\left(X^{\prime}, f^{\prime}\right)$ is an upper bound 
for $\left\{\left(X_{i}, f_{i}\right): i \in I\right\}$ then for each $i \in I, b_{i} X \leqq b X=\beta X$ and so $a X \leqq b X$. Then $b \leqq a$ and so $\left(X_{a}^{*}, f_{a}^{*}\right) \leqq\left(X^{\prime}, f^{\prime}\right)$. Thus the compactifications of $f$ form a complete upper semilattice.

This semilattice will be a complete lattice if and only if it has a smallest member.

Now, if there is a smallest compactification of $f$, determined by the compactification $s X$ of $X$ say, where $s \leqq u$, then if $s\left|f^{-1}(Y) \backslash X \neq u\right| f^{-1}(Y) \backslash X$ there exist points $x, y$ in $f^{-1}(Y) \backslash X$ belonging to the same block in $u$ but belonging to different blocks in $s$. But then the partition $m$ produced from $s$ by joining the blocks containing $x$ and $y$ is again u.s.c. and is strictly less than $s$ on $f^{-1}(Y)$. It follows from Corollary 3 that $\left(X_{m}^{*}, f_{m}^{*}\right)<\left(X_{s}^{*}, f_{s}^{*}\right)$ contradicting the fact that $\left(X_{s}^{*}, f_{s}^{*}\right)$ is minimal.

Conversely, if $s X$ is such that $s\left|f^{-1}(Y) \backslash X=u\right| f^{-1}(Y) \backslash X$ then for any compactification $a X$ of $X, a \wedge u \leqq u$ and so from Corollary 3

$$
\left(X_{s}^{*}, f_{s}^{*}\right) \leqq\left(X_{a}^{*}, f_{a}^{*}\right) \text {. }
$$

In particular if $X$ is locally compact the compactifications of $f$ form a complete lattice, since in this case the one-point compactification of $X$ satisfies the requirements of $s X$ in Corollary 5 .

\section{REFERENCES}

(1) G. L. Cain, JR., Compactification of Mappings, Proc. Amer. Math. Soc. 23 (1969), 298-303.

(2) G. L. CAIN, JR., Extensions and compactifications of mappings, Math. Ann. 191 (1971), 333-336.

(3) G. L. CAIN, JR., Metrizable Mapping compactifications, General Topology and Appl. 2 (1972), 271-275.

(4) P. A. Firby, Lattices and compactifications I, Proc. London Math. Soc. (3) 27 (1973), 22-50.

(5) P. A. Firgy, Lattices and compactifications II, Proc. London Math. Soc. (3) 27 (1973), 51-60.

(6) O. NJASTAD, A note on compactification by bounding systems, J. London Math. Soc. 40 (1965), 526-532.

(7) G. T. Whyburn, A unified space for mappings, Trans. Amer. Math. Soc. 74 (1953), 344-350.

\section{THE UNIVERSITY}

\section{EXETER}

